\title{
EXPRESSION DE LA TENSION PHOTOVOLTAÏQUE INDUITE PAR TRANSITION INTERBANDE DANS DES COUCHES MINCES DE SULFURE DE ZINC
}

\author{
A. TOSSER et H. MURRAY \\ Laboratoire d'Electronique et d'Automatique, U.E.R. des Sciences et Techniques, B.P. 4006, \\ 76077 - Le Havre Cédex, France
}

(Received March 18, 1975; in final form April 8, 1975)

\begin{abstract}
En considérant les variations dimensionnelles de la tension photovoltaïque induite en circuit ouvert par des rayonnements lumineux d'énergie supérieure à $3,66 \mathrm{eV}$ dans des structures sandwich $\mathrm{Al} / \mathrm{ZnS} / \mathrm{Au}$, ainsi que les variations avec le flux lumineux du courant de court-circuit et de la conductance correspondant à la phototransition entre bandes, l'hypothèse d'une quasi-bande de conduction créée par l'ionisation de centres coulombiens est avancée. Elle conduit à une formulation de la tension photovoltaïque en bonne cohérence avec l'expérience.
\end{abstract}

Variations with thickness of open-circuit voltage, short-circuit current and photoconductance associated to interband transition lead to postulate the existence of a quasi-conduction band, associated with ionized coulombic centres, in $\mathrm{Al} / \mathrm{ZnS} / \mathrm{Au}$ illuminated (photon energy $>3,66 \mathrm{eV}$ ) sandwich structures. An expression of open-circuit voltage is deduced, in good agreement with experimental data.

\section{INTRODUCTION}

Les effets photovoltaiques qui apparaissent dans des structures "sandwich" en couches minces du type $\mathrm{Al} / \mathrm{ZnS} / \mathrm{Au}$, préparées par pulvérisation cathodique réactive diode, ont déjà été présentés. ${ }^{1}$ Nous nous proposons, dans cet article, de fournir quelques indications complémentaires relatives au niveau de la tension photovoltaique induite par un rayonnement lumineux d'énergie supérieure à $3,66 \mathrm{eV}$.

\section{RÉSULTATS EXPÉRIMENTAUX ANTÉRIEURS}

Nous adoptons les conventions de signes suivantes:

\section{Convention 1}

Le courant qui traverse la structure est positif s'il correspond à un transfert d'électrons de l'électrode d'aluminium vers la contre-électrode d'or, à travers le sulfure de zinc.

\section{Convention 2}

Le potentiel de l'électrode d'aluminium est choisi comme référence des potentiels Avec ces con- ventions de signe, si $V$ désigne la tension électrique appliquée sur l'électrode d'or, si I désigne le courant traversant la structure, si $V_{\text {pho }}$ désigne la tension photovoltaique en circuit ouvert, et si $I_{\text {pho }}$ est le courant de court-circuit photoinduit, nous avons établi ${ }^{1}$ expérimentalement les relations suivantes, valables pour des épaisseurs de diélectrique comprises entre 260 et $540 \AA$

$$
\begin{aligned}
& I=\left(G_{\mathrm{ph}}+G_{0}\right)\left(V-V_{\mathrm{pho}}\right) \\
& I_{\mathrm{pho}}=-\left(G_{\mathrm{ph}}+G_{0}\right) V_{\mathrm{pho}}
\end{aligned}
$$

$I_{\text {pho }}$ étant de signe négatif et $V_{\text {pho }}$ étant de signe positif; la conductance $G_{\mathrm{ph}}$ est relative au mouvement des électrons dans la bande de conduction du diélectrique ${ }^{1}$ dont nous avons montré qu'elle est due à une transition interbande photoinduite, ${ }^{2}$ qui laisse des trous dans la bande de valence, et la conductance $G_{0}$ étant relative à une conduction du type PooleFrenkel se produisant, par l'intermédiaire des centres coulombiens du diélectrique, pour une faible valeur du décalage des niveaux de Fermi des électrodes. ${ }^{1}$

Des relevés expérimentaux ${ }^{1}$ ont montré que la conductance $G_{\mathrm{ph}}$ était proportionnelle au flux lumineux. F, comme le courant de court-circuit $I_{\mathrm{pho}}$ et que la conductance $G_{0}$ ne dépendait pas du flux.

L'équation (2) rend compte de l'annulation simultanée $^{1}$ du courant de court-circuit et de la tension 
photovoltaique en circuit ouvert dans tout le domaine des énergies supérieures à, $3,66 \mathrm{eV}$.

\section{INTERPRÉTATION QUALITATIVE DES LOIS DE VARIATION DE LA TENSION PHOTOVOLTAÏQUE}

\subsection{Existence d'une Quasi-Bande de Conduction}

L'expression du courant de court-circuit $I_{\text {pho }}$ montre que les effets Schottky qui se produisent sur les électrodes ne donnent pas lieu à une circulation de courant; on peut d'ailleurs avancer que les électrons photoexcités de l'électrode d'aluminium ne peuvent se déplacer contre le champ électrique interne, tous les auteurs ${ }^{3}$ s'accordant à reconnaître que le potentiel de sortie de l'or est plus élevé que celui de l'aluminium: en ce qui concerne les photoélectrons issus de la contre-électrode d'or, nous postulons que certains disparaissent rapidement au voisinage de la contre-électrode soit par capture dans un centre coulombien, soit, plus vraisemblablement, par recombinaison avec les trous qui ont tendance à s'accumuler à l'interface $\mathrm{ZnS} / \mathrm{Au}$; dans ces hypothèses, le seul courant photoélectrique $I_{\mathrm{ph}}$ correspondant à une énergie de la bande de conduction du $\mathrm{ZnS}$ est du à la migration des électrons de valence photoexcités sous l'action du champ électrique interne, modifié par la tension $V$ existant entre les électrodes; en court-circuit, ce courant correspond à un déplacement d'électrons de l'électrode d'or vers l'électrode d'aluminium. Il s'écrit, en désignant par $V_{0}$ la différence de potentiel qui correspond au champ électrique interne apparent à circuit ouvert, soit, si on pose

$$
V_{0}=\left(\varphi_{\mathrm{Au}}-\varphi_{\mathrm{A} 1}\right) / q
$$

$q$ étant la charge de l'électron

$\varphi_{\mathrm{Au}}$ et $\varphi_{\mathrm{A} 1}$ étant les potentiels de sortie relatifs apparents de l'or et de l'aluminium dans la structure étudiée.

$$
I_{\mathrm{ph}}=G_{\mathrm{ph}}\left(V-V_{0}\right)
$$

Lorsqu'une tension $V$ est appliquée sur la contreélectrode d'or, un courant Poole-Frenkel $I_{\mathrm{pF}}$ circule: les électrons se dirigent vers l'électrode d'or si la tension $V$ est positive; ce courant $I_{\mathrm{pF}}$ est fonction linéaire de $V$, à faible niveau soit

$$
I_{\mathrm{pF}}=G_{0} V
$$

Le courant I total traversant la structure illuminée a alors pour expression:

d'où

$$
I=I_{\mathrm{pF}}-I_{\mathrm{ph}}
$$

$$
I=G_{0} V-G_{\mathrm{ph}}\left(V_{0}-V\right)
$$

$G_{\mathrm{ph}}$ et $G_{0}$ étant les conductances définies au paragraphe précédent, nous sommes ainsi amenés à poser, $F$ étant la valeur du flux lumineux:

$$
G_{\mathrm{ph}}=F \cdot g_{\mathrm{ph}}
$$

L'expression de la conductance $G_{\mathrm{ph}}$ ainsi définie est bien compatible avec la définition proposée antérieurement $^{1}$ à partir des observations expérimentales.

En circuit ouvert le courant I est nul et la tension $V$ est la tension photovoltaïque $V_{\text {pho }}$. L'équation (4) s'écrit sous la forme $\left(4^{\prime}\right)$;

$$
0=G_{0} V_{\text {pho }}-G_{\mathrm{ph}}\left(V_{0}-V_{\mathrm{pho}}\right)
$$

d'où

$$
V_{\mathrm{pho}}=\frac{G_{\mathrm{ph}}}{G_{\mathrm{ph}}+G_{0}} V_{0}
$$

La variation de cette tension photovoltaique en fonction du flux $\mathrm{F}$ nous est aussi connue par les mesures expérimentales (Figure 1).

La relation $\left(4^{\prime}\right)$ permet d'écrire l'expression de $I$ ous la forme

$$
I=\left(G_{\mathrm{ph}}+G_{0}\right)\left(V-V_{\mathrm{pho}}\right)
$$

qui est bien la relation déduite des relevés expérimentaux (équation 1) à partir de laquelle a été déterminée la variation de la quantité $\left(G_{\mathrm{ph}}+G_{0}\right)$ en fonction du flux $F$ (Figure 1).

En court-circuit le courant photoinduit vaut

$$
I_{\text {pho }}=-G_{\text {pho }} \cdot V_{0} \text {. }
$$

ou encore en tenant compte de la relation (5)

$$
I_{\mathrm{pho}}=-\left(G_{\mathrm{ph}}+G_{0}\right) V_{\mathrm{pho}}
$$

ce qui est exactement la loi expérimentale (Eq. 2).

L'observation de la variation linéaire du courant $I_{\mathrm{pho}}$ avec le flux $F^{1}$ nous conduit d'aprés l'équation $\left(4^{\prime}\right)$ à postuler que la valeur de $V_{0}$ est indépendante du flux lumineux. La Figure 2 fournit la variation de la quantité

$$
\frac{G_{\mathrm{ph}}+G_{0}}{G_{\mathrm{ph}}} \cdot V_{\mathrm{pho}}=V_{0}
$$

en fonction du flux $F$, pour une épaisseur diélectrique égale à $450 \AA$ et une lumière de $3100 \AA$ de longueur d'onde; cette variation est déterminée à partir des relevés expérimentaux de la Figure 1. 


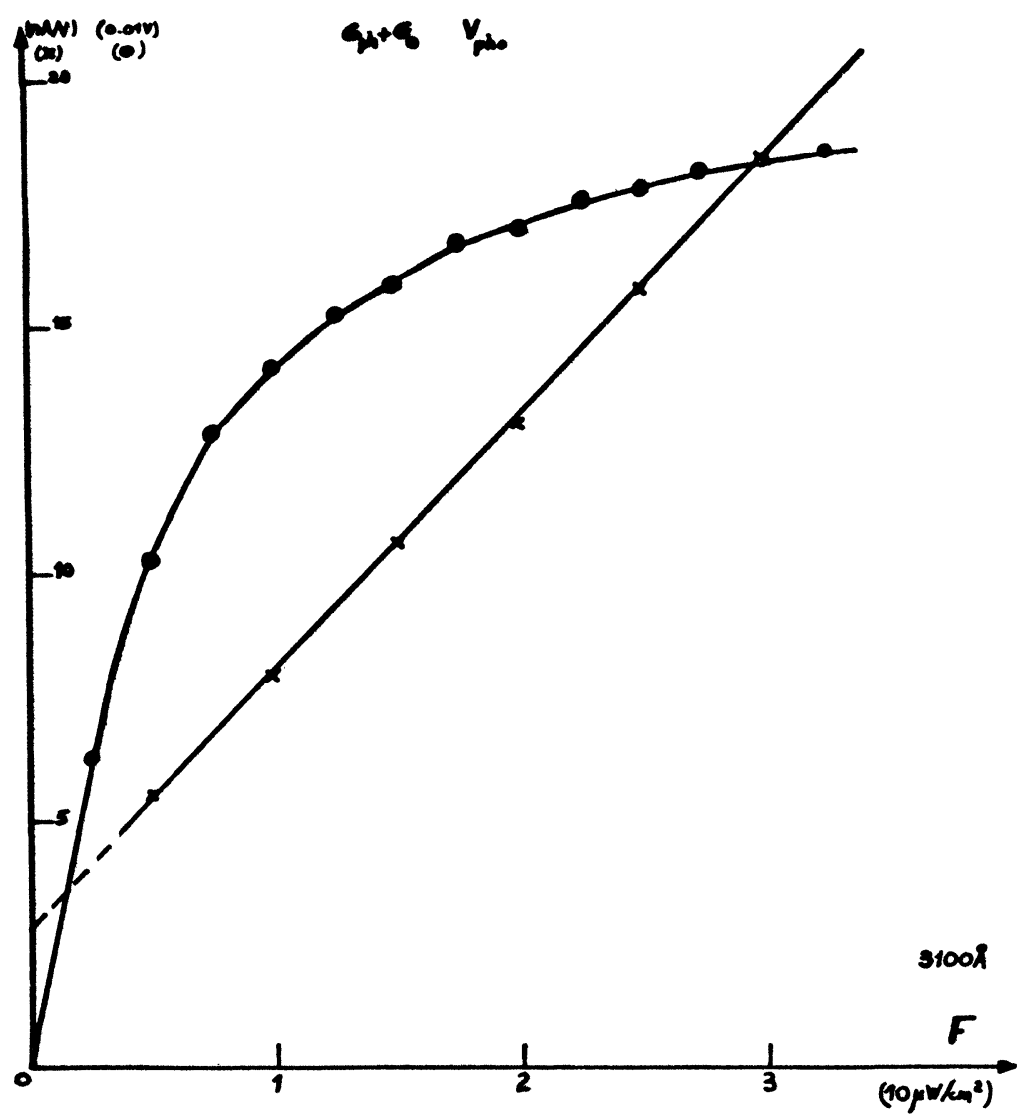

FIGURE 1 Variations avec le flux lumineux $F$ de la somme des conductances photoélectriques $\left(G_{\mathrm{ph}}+G_{0}\right)$ et de

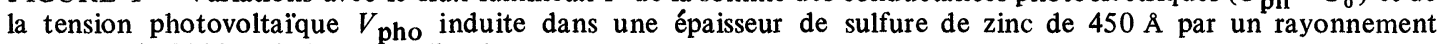
lumineux de 3100 \& de longueur d'onde.

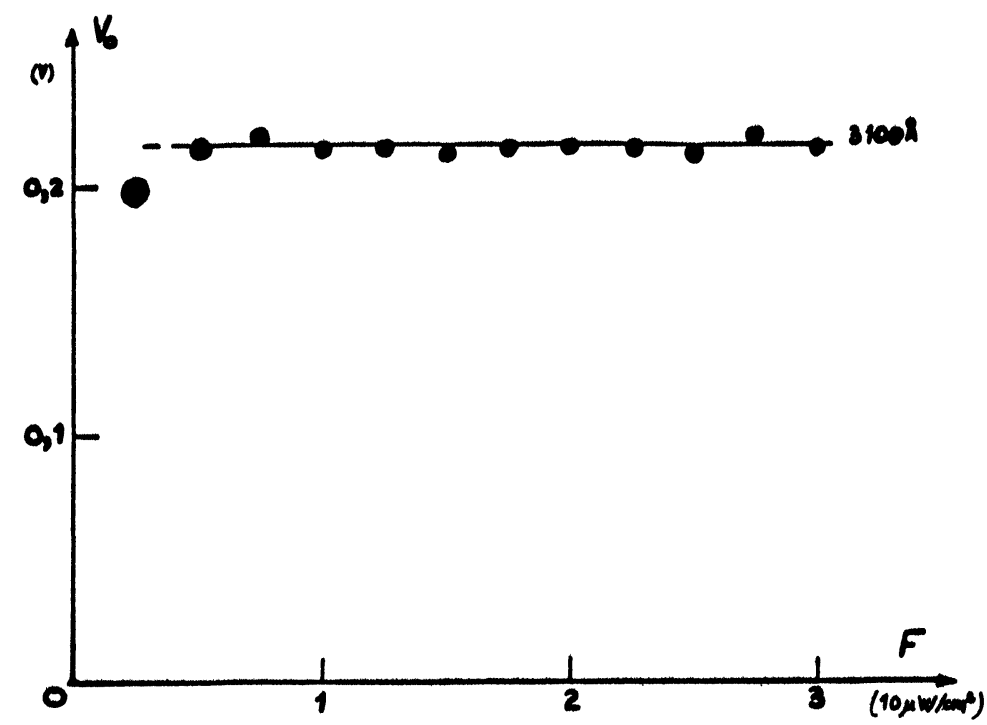

FIGURE 2 Variations avec le flux lumineux $F$ de $3100 \AA$ de longueur d'onde de la différence des potentiels de sor tie relatifs apparents des électrodes (Al et $\mathrm{Au}$ ). 
Les valeurs obtenues sont variables avec l'épaisseur de diélectrique et la longueur d'onde de la lumière; elles sont trop faibles pour correspondre à la différence des travaux de sortie de l'or et de l'aluminium, ${ }^{3}$ et ne peuvent qu'être dues à une perturbation; de plus, l'invariance de $V_{0}$ avec le flux n'est observée que dans un domaine d'épaisseur de diélectrique comprise entre 260 et $460 \AA$.

L'existence de centres coulombiens dans le diélectrique ${ }^{4}$ nous conduit à supposer que le rayonnement lumineux, en chassant des électrons de ces centres, leur permet d'acquérir un potentiel d'ionisation, qui intervient dans le processus de conduction Poole-Frenkel entre les électrodes, par sauts de centre à centre ${ }^{5}$ et qui détermine un abaissement local de la barrière de potentiel, sous réserve qu'il y ait localement recouvrement des abaissements de barrière, soit entre centres coulombiens voisins, soit à une interface, le champ électrique interne en circuit ouvert se trouve modifié.

Comme le rayonnement lumineux permet des transitions au-dessus de la bande interdite du diélectrique, ${ }^{2}$, nous postulons que les centres coulombiens ne conservent que la population électronique nécessaire à l'équilibre électrostatique en circuit ouvert, conjointement déterminé par les effets Poole-Frenkel et les phototransitions; le taux de dépopulation ainsi que le potentiel d'ionisation atteignent donc une valeur limite, indépendante de la valeur du flux lumineux pour la longueur d'onde considérée et pour une épaisseur donnée du diélectrique; ainsi peut être comprise l'indépendance de la conductance $G_{0}$ par rapport au flux lumineux. A cette valeur limite du potentiel d'ionisation indépendante du flux, correspond un abaissement local déterminé de la barrière de potentiel, ce qui permet l'apparition d'un champ électrique interne caractérisant chaque longueur d'onde du rayonnement lumineux et correspondant à un équilibre électrostatique qui dépend de l'épaisseur du diélectrique.

\subsection{Phénomènes Dimensionnels}

Pour une longueur d'onde donnée, nous observons une forte croissance de la tension photovoltaique en fonction de l'épaisseur (Figure 3), ce qui confirme que, dans un vaste domaine d'épaisseur, la bande de conduction est fortement perturbée par l'ionisation des centres coulombiens, car l'abaissement de barrière est d'autant moins marqué que le champ électrique interne intrinsèque est plus faible, c'est-à-dire que l'épaisseur est plus élevée.

Nous pouvons ainsi préciser l'hypothèse avancée plus haut: l'ionisation des centres coulombiens produit des abaissements de barrière qui, par recouvrement entre eux ou avec l'abaissement de

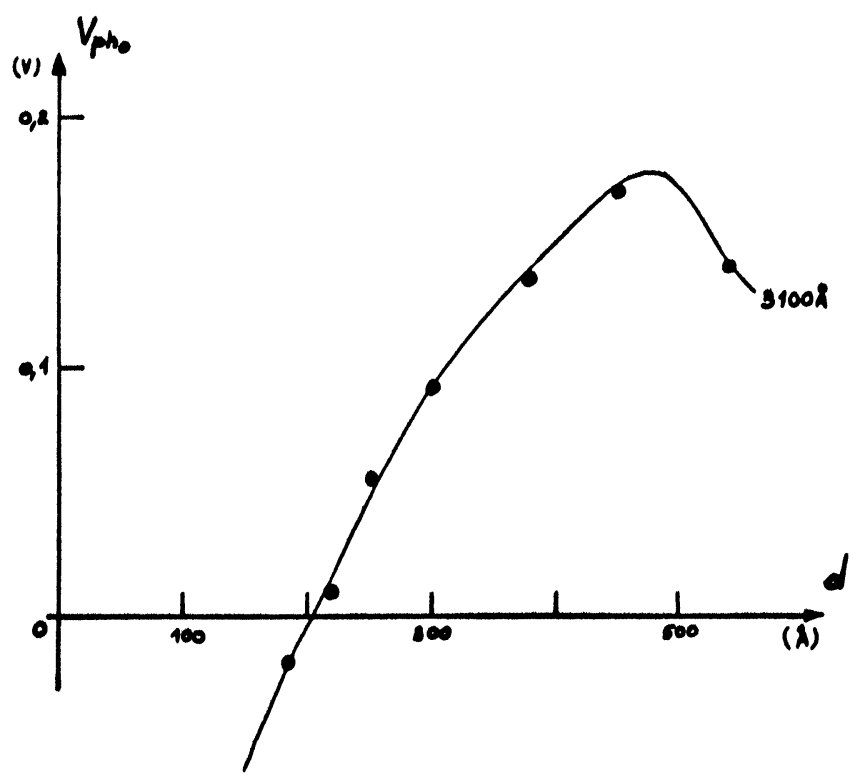

FIGURE 3 Variations de la tension photovoltaïque $V_{\text {pho }}$ induite par la lumière de $3100 \AA$ de longueur d'onde, et de flux égal à $20 \mu \mathrm{w} / \mathrm{cm}^{2}$ dans une couche de sulfure de zinc en fonction de l'épaisseur $d$. 
force-image de l'interface $\mathrm{ZnS} / \mathrm{Au}^{6}$ provoquent l'apparition locale d'une quasi-bande de conduction qui détermine la conduction des électrons photoexcités. Nous pouvons alors postuler qu'il se produit un renversement du champ interne à l'interface $\mathrm{ZnS} / \mathrm{Au}$ (où le flux lumineux est maximal), ce qui interdit toute émission Schottky à partir de l'électrode d'or en direction de l'électrode de base et complète l'hypothèse du $\S 3.1$.

L'équation (4) peut.se mettre sous la forme

$$
I=\left(G_{0}+G_{\mathrm{ph}}\right) V-G_{\mathrm{ph}} V_{0}
$$

Le relevé expérimental des variations du courant $I$ en fonction de la tension $V$ qui conduit bien à une loi linéaire, ${ }^{1}$ dans un large domaine de tensions (s'étendant de $-0,3 V$ à $+0,6 V$, lorsque l'épaisseur de diélectrique vaut $240 \AA$ ) et dans un domaine d'épaisseurs compris entre 190 et $540 \AA$ (Figure 4)

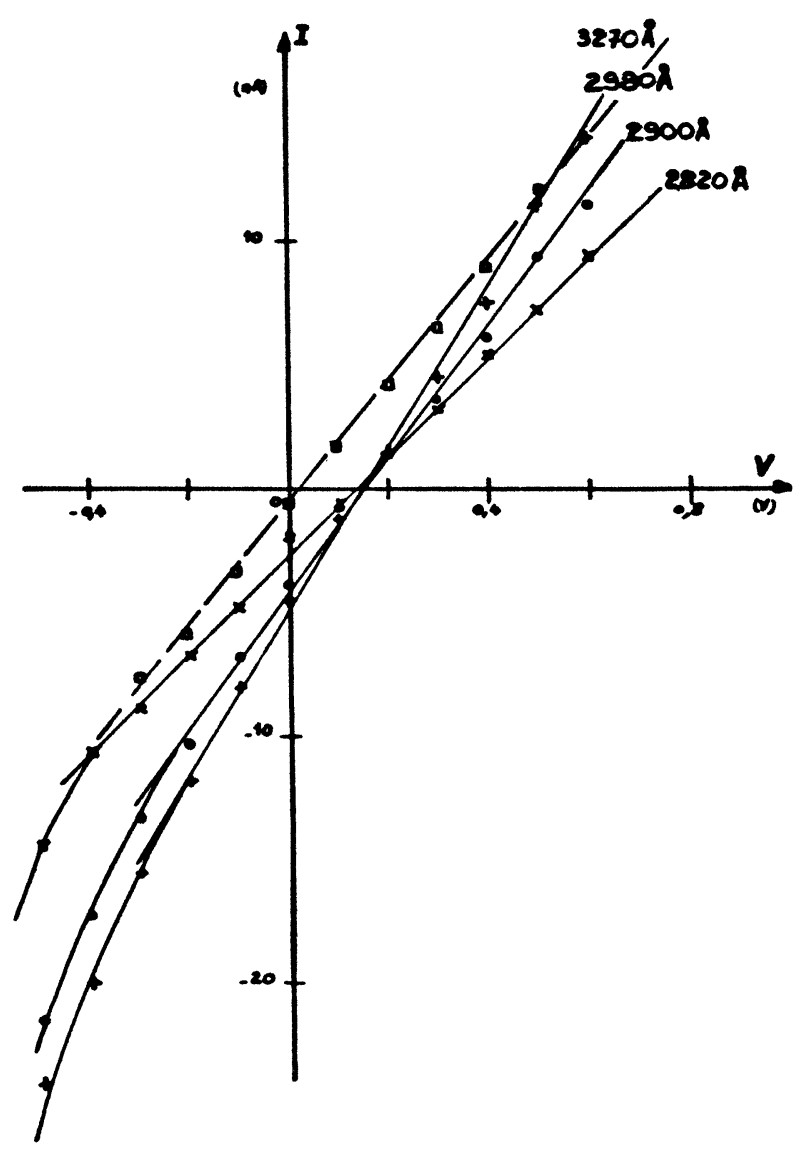

FIGURE 4 Variations du courant photoinduit I par une lumiere de longueur d'onde variable (indiquée en paramètre) dans une épaisseur sandwich de $240 \AA$ de sulfure de zinc en fonction de la différence de potentiel $V$ appliquée sur l'électrode supérieure. témoigne de l'invariabilité de la tension $V_{0}$ et de la conductance $G_{0}$ par rapport à la tension $V$.

Nous sommes ainsi conduits à postuler que les variations des raccordements aux interfaces sous l'effet du décalage des niveaux de Fermi des métaux des électrodes n'interviennent pas d'une facon appréciable sur la position de la quasibande de conduction dans la zone où apparaissent la majorité des photoélectrons de conduction; il paraît en effet raisonnable de supposer que la conduction PooleFrenkel entre le centre coulombien le plus proche de l'interface $\mathrm{ZnS} / \mathrm{Au}$ et la contre-électrode d'or a pour objet d'ajuster automatiquement le niveau d'ionisation en fonction de la position du niveau de Fermi de l'or.

Pour des valeurs négatives de la tension situées en dehors du domaine précédent, le courant I croît rapidement comme un courant d'émission Schottky; cette variation nous paraît indiquer que le champ interne apparent est bien dû à une distorsion se produisant à l'interface $\mathrm{ZnS} / \mathrm{Au}$ où le flux est maximal.

Comme le potentiel d'ionisation des centres coulombiens, qui est indépendant du flux lumineux, provoque un abaissement de barrière dépendant de la racine carrée du champ électrique initial, nous postulons que la tension $V_{0}$ peut s'écrire, par transformation de l'équation $\left(3^{\prime}\right)$ :

$$
V_{0}=\left[\Delta \Psi-H(\lambda) \cdot d^{-1 / 2}\right] \cdot q^{-1}
$$

$d$ étant l'épaisseur du diélectrique, $q$ la charge de l'électron et $\Delta \Psi$ une différence de travaux de sortie, indépendante des effets Poole-Frenkel. La tension photovoltaique a alors pour expression, d'après (5)

$V_{\text {pho }}=\left\{G_{\mathrm{ph}} /\left(G_{\mathrm{ph}}+G_{0}\right)\right\} \cdot\left[\Delta \Psi-H(\lambda) \cdot d^{-1 / 2}\right] \cdot q^{-1}$

Nous observons effectivement, pour une longueur d'onde donnée, et un niveau déterminé de flux lumineux, une variation de la tension photovoltaique $V_{\text {pho }}$ en fonction de la racine carrée de l'épaisseur qui est bien conforme à cette loi (Figures 5 et 6). On note de plus que, si l'épaisseur de diélectrique est comprise entre 260 et $460 \AA$, le facteur $G_{\mathrm{ph}} /\left(G_{\mathrm{ph}}\right.$ $\left.+G_{0}\right)$ ne dépend pas de $d$, ce qui est compatible avec les Equations ( $\left.4^{\prime}\right)$ et ( $\left.4^{\prime \prime}\right)$ si les conductances $G_{\mathrm{ph}}$ et $G_{0}$ ont même variation dimensionnelle; cette hypothèse nous paraît acceptable, étant donnée l'origine photoélectrique commune des deux phénomènes.

En utilisant ces équations, l'expression de la tension photovoltaique induite en circuit ouvert $V_{\text {pho }}(\lambda, F)$ est, lorsque l'épaisseur $d$ du diélectrique est comprise entre 240 et $460 \AA$ et la longueur d'onde 

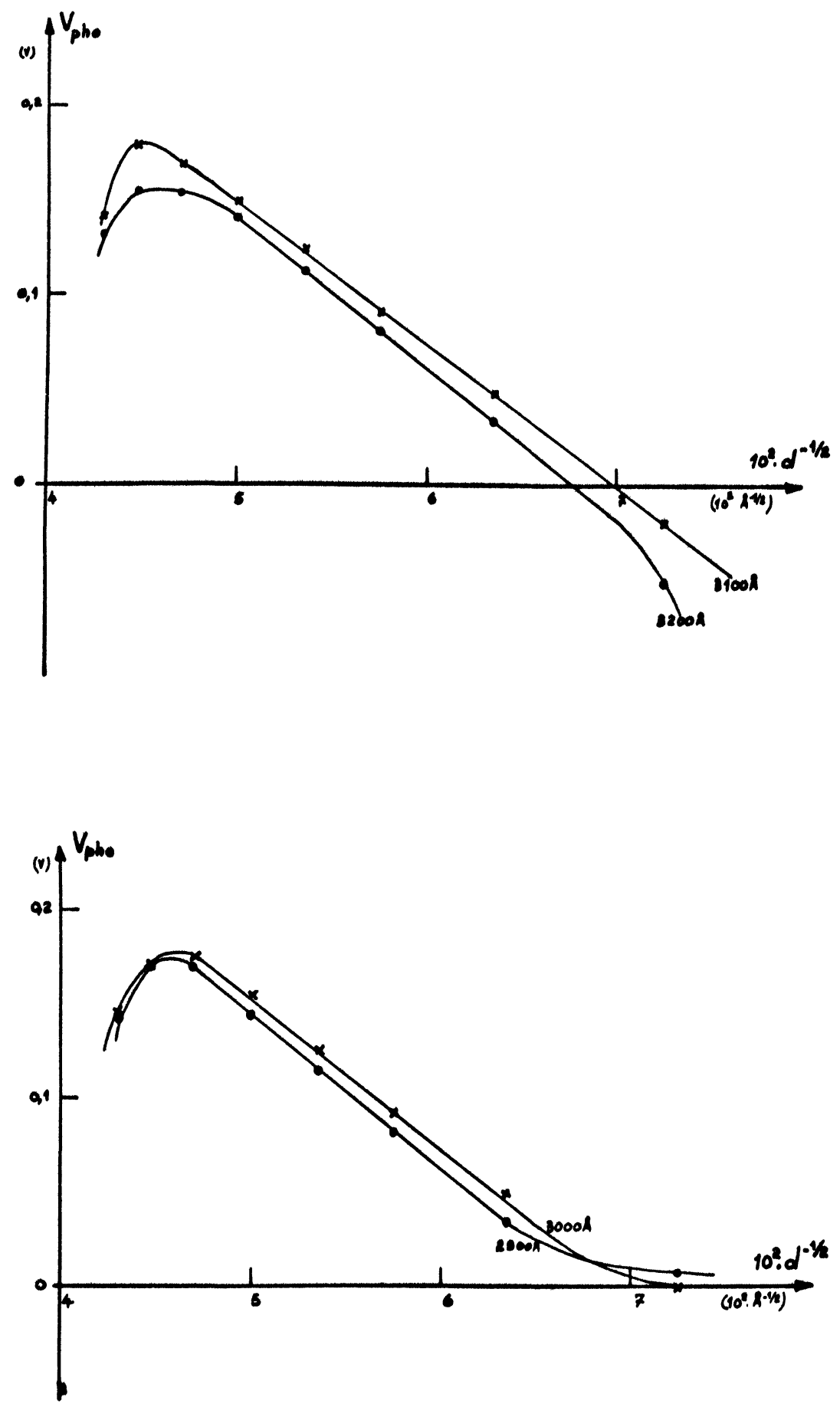

FIGURE 5 et 6 Variations de la tension photovoltaique $V_{\text {pho }}$ (indiquée en paramètre) induite par une lumière de longueur d'onde variable dans une couche de sulfure de zinc d'épaisseur $d$ en fonction de $10^{2} \cdot d^{-1 / 2}$. 
$\lambda$ du rayonnement lumineux, de flux $F$, comprise entre 2800 et $3300 \AA$ :

$$
\begin{aligned}
V_{\mathrm{pho}}(\lambda, F)=\{F \cdot & \left.g_{\mathrm{ph}}(\lambda) /\left[F \cdot g_{\mathrm{ph}}(\lambda)+g_{0}(\lambda)\right]\right\} \\
\cdot & {\left[\Delta \psi-H(\lambda) d^{-1 / 2}\right] \cdot q^{-1} }
\end{aligned}
$$

Les fonctions $g_{\mathbf{p h}}(\lambda)$ et $H(\lambda)$ sont lentement variables avec $\lambda$ et indépendantes du flux lumineux $F$; la quantité $\Delta \Psi$ est voisine de $0,67 \mathrm{eV}$ pour les structures $\mathrm{Al} / \mathrm{ZnS} / \mathrm{Au}$.

Cette loi n'a plus de validité lorsque l'épaisseur est inférieure à $240 \AA$; on peut penser en effet que le champ électrique interne est perturbé dans des conditions différentes par suite de la forte densité apparente des centres coulombiens de la zone critique, qui est 4 à 5 fois plus importante que dans le reste de l'épaisseur. ${ }^{7}$ Nous aurons l'occasion ultérieurement de préciser quelques propriétés de cette zone critique, de moins de $150 \AA$ d'épaisseur, ${ }^{7}$ qui est caractéristique des couches minces pulvérisées. ${ }^{8}$

\section{ESSAI D'INTERPRÉTATION THÉORIQUE}

Considérons le schéma de bande de la structure $\mathrm{Al} / \mathrm{ZnS} / \mathrm{Au}$ (Figure 7). Nous savons que le diélectrique comporte une répartition régulière de centres coulombiens $(4,1)$ qui permet, par cumul d'abaissements locaux de barrière, un renversement du champ électrique interne ( $\S 3)$.

Désignons par $d$ l'épaisseur de diélectrique

$d_{1}$ l'épaisseur de diélectrique où le champ électrique, en circuit ouvert, est inversé

$l$ la longueur d'affaiblissement du rayonnement lumineux de longueur d'onde $q \cdot V_{\mathrm{s}}=\phi_{\mathrm{Au}}-\phi_{\mathrm{A} 1}$ la différence des potentiels relatifs de sortie de l'or et de l'aluminium

$q \cdot V$ le décalage des niveaux de Fermi des métaux introduit par une tension $V$ appliquée sur l'électrode d'or

$n(x)$ la densité de photoélectrons injectés dans la bande de conduction, à la distance $\mathrm{x}$ de l'interface $\mathrm{ZnS} / \mathrm{Au}$

$F$ la valeur du flux lumineux

$I_{\mathrm{ph}}$ le courant photoélectrique correspondant aux mouvements des photoélectrons dans la bande de conduction du diélectrique $\sigma$ la conductivité des photoélectrons

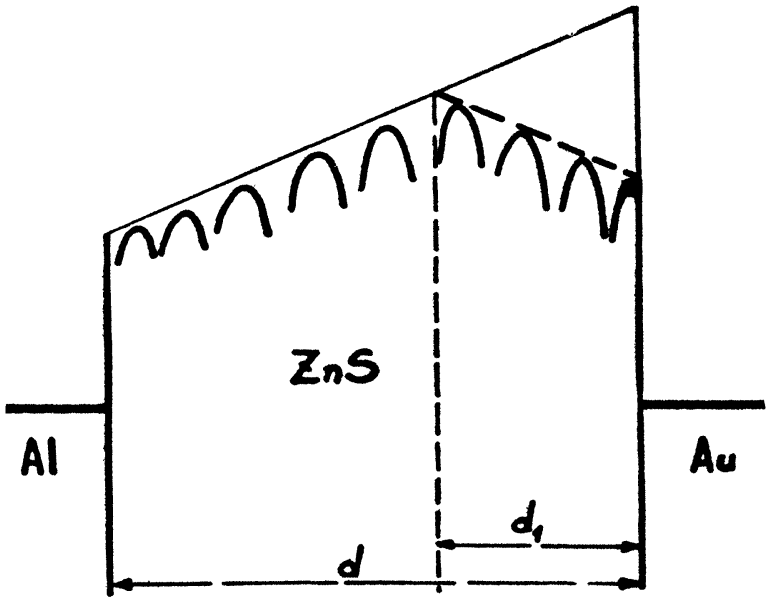

FIGURE 7 Modèle de bandes simplifié des couches de sulfure de zinc: dans l'épaisseur $d_{1}$ la quasi-bande de conduction est inversée par rapport à la bande de conduction habituelle, qui est supposée pratiquement inchangée dans le reste du domaine d'épaisseur.

dans la bande de conduction du sulfure

$q \cdot \delta V_{1}$ l'abaissement de la barrière à l'interface $\mathrm{ZnS} / \mathrm{Au}$

La densité $n(x)$ peut s'écrire, en admettant une absorption exponentielle de la lumière dans le sulfure:

$$
n(x)=n \cdot F \cdot \exp (-x / 1)
$$

Nous postulons que le décalage des niveaux de Fermi produit un champ électrique se superposant aux champs préexistants, supposés de valeurs constantes dans les deux domaines de la couche de sulfure; dans le premier domaine, d'épaisseur $d_{1}$ au voisinage de la contre-électrode d'or le champ est inversé et dans le deuxième, d'épaisseur $d-d_{1}$, le champ a le sens du champ intrinsèque; dans ces hypothèses, le courant photoinduit $I_{\mathrm{ph}}$, positif lorsqu'il correspond à un transfert d'électrons en direction de l'électrode d'or, est la somme des courants partiels de signes opposés circulant dans chaque domaine, soit

$$
\begin{aligned}
I_{\mathrm{ph}} \approx \sigma \cdot N_{1}\left[d^{-1} \cdot\left(-V_{\mathrm{s}}+V\right)+d_{1}^{-1} \cdot\left(\delta V_{1}\right)\right] & \\
& -\sigma \cdot N_{2} \cdot d^{-1}\left(V_{\mathrm{s}}-V\right)
\end{aligned}
$$

Les nombres $N$ correspondent au nombre total de photoélectrons dans chaque domaine. 
D'où

$I_{\mathrm{ph}} \approx \sigma\left(N_{1}+N_{2}\right)\left[d^{-1}\left(V-V_{\mathrm{s}}\right)\right.$

$$
\left.+\left\{N_{1} /\left(N_{1}+N_{2}\right)\right\} \cdot d_{1}^{-1} \cdot \delta V_{1}\right]
$$

Le nombre de photoélectrons injectés dans la partie de la bande de conduction du sulfure dont la pente est inversée vaut, d'après (9)

$$
N_{1}=n . F \cdot \int_{0}^{d_{1}} \exp (-x / l) \cdot d x
$$

Soit

$N_{1}=\mathrm{n} \cdot \mathrm{F} \cdot 1 \cdot\left(1-\exp \left(-d_{1} / l\right)\right.$

Et de même

$$
N_{1}+N_{2}=\mathrm{n} \cdot \mathrm{F} \cdot 1(1-\exp (-d / l))
$$

En tenant compte des relations (11) et (12) pour transformer l'équation (10), l'expression de $I_{\mathrm{ph}}$ peut s'écrire sous la forme déjà proposée

$$
\begin{gathered}
I_{\mathrm{ph}}=G_{\mathrm{ph}}\left(V-V_{0}\right) \quad \text { avec } \\
G_{\mathrm{ph}} \approx \frac{\sigma n l F}{d}(1-\exp (-d / l)) \\
V_{0} \approx V_{\mathrm{s}}-\frac{1-\exp \left(-d_{1} / l\right)}{1-\exp (-d / l)} \cdot \frac{d}{d_{1}} \cdot \delta V_{1}
\end{gathered}
$$

Lorsque l'épaisseur $d$ de diélectrique est faible devant la longueur d'affaiblissement $l$, ces équations deviennent

et

$$
G_{\mathrm{ph}} \approx \sigma \cdot \mathrm{n} \cdot \mathrm{F}
$$

$$
V_{0} \approx V_{\mathrm{s}}-\delta V_{1}
$$

Lorsque l'épaisseur $d_{1}$ est faible devant la longueur 1 , nous écrivons

$$
\begin{gathered}
G_{\mathrm{ph}} \approx \sigma n F \cdot[1-\exp (-d / l)] /(d / l) \\
V_{0} \approx V_{\mathrm{s}}-\{(d / l) /[1-\exp (-d / l)]\} \delta V_{1}
\end{gathered}
$$

Afin d'obtenir une formulation plus commode pour les calculs, nous nous proposons d'effectuer une détermination approchée de l'expression de la fonction

$$
g(d / l)=(d / l) /[1-\exp (-d / l)],
$$

lorsque l'épaisseur $d$ n'est pas négligeable devant 1 .

Considérons la fonction $g(u)$ de la variable $u=d / l$ :

$$
g(u)=u /(1-\exp (-u))
$$

Posons

$u=u_{0}+h$

avec $h$ petit devant $u_{0}$, et

$$
\alpha=e^{-u_{0}},
$$

l'équation (15) s'écrit, après développement limité, et en choisissant $u_{0}$ tel que

$$
\begin{gathered}
g\left(u_{0}\right) \approx 1 \\
g\left(u_{0}+h\right) \approx 1+\frac{h}{2},
\end{gathered}
$$

$h$ étant le quotient par $l$ de la différence entre l'épaisseur $d$ et une épaisseur $d_{0}$, petite devant $l$ et pour laquelle les formules (13') et (14') sont valables.

L'expression (14") devient alors

$$
V_{0} \approx V_{\mathrm{s}}-\left(1+\frac{h}{2}\right) \delta V_{1}
$$

et l'expression (13"')

$$
G_{\mathrm{ph}} \approx \sigma n F\left(1-\frac{h}{2}\right)
$$

Pour l'ensemble des formules approchées déduites des Équations (13) et (14), la quantité $\delta V_{1}$ est l'abaissement de barrière réalisé à l'interface $\mathrm{ZnS} / \mathrm{Au}$ dont la valeur est proportionnelle à la racine carrée du champ électrique intrinsèque, ce qui permet de postuler une variation comme $d^{-1 / 2}$. la remarque du $\$ 3.1$ peut être reprise pour justifier l'indépendance de $\delta V_{1}$ par rapport au flux lumineux. Ces considérations permettent d'écrire

$$
\delta V_{1}=H_{1}(\lambda) \cdot d^{-1 / 2}
$$

la quantité $H_{1}(\lambda)$ étant variable avec la longueur d'onde comme l'absorption de la lumière dans le diélectrique.

Si nous supposons que l'épaisseur du diélectrique est petite devant la longueur d'absorption $l$ de la lumière dans le sulfure, l'équation (5) s'écrit, d'après les formules $\left(13^{\prime}\right)\left(14^{\prime}\right)$ et (18)

$$
V_{\text {pho }} \approx\left\{\sigma n F /\left(\sigma n F+G_{0}\right)\right\} \cdot\left[V_{\mathrm{s}}-H_{1}(\lambda) \cdot d^{-1 / 2}\right]
$$

Nous retrouvons la formule déduite des expérimentations (équation (8)), à condition de poser

$$
V_{\mathrm{s}}=\Delta \psi \cdot q^{-1}
$$

Un écart existe cependant entre la valeur expéri- 
mentale de $\Delta \Psi(0,665 \mathrm{eV})$ et la valeur théorique égale à la différence des travaux de sortie de l'aluminium et de l'or (au moins $0,8 \mathrm{eV})^{3}$ Cette divergence nous paraît pouvoir être attribué à des phénomènes à l'interface $\mathrm{Al} / \mathrm{ZnS}$ déjà mis en évidence pour les interfaces $\mathrm{Al} / \mathrm{Al}_{2} \quad \mathrm{O}_{3}{ }^{9-11}$ et $\mathrm{Al} / \mathrm{Ca} \mathrm{WO}{ }_{4} .^{9}$

Lorsque l'épaisseur de la zone d'inversion du champ électrique interne est faible devant la longueur d'affaiblissement de la lumière, l'expression de la tension photovoltaique en circuit ouvert devient, en utilisant les Équations (5), (13"') et $\left(14^{\prime \prime \prime}\right)$

$$
\begin{gathered}
V_{\mathrm{pho}} \approx\left\{\sigma n F\left(1-\frac{h}{2} /\left[\sigma n F\left(1-\frac{h}{2}\right)+G_{0}\right]\right\}\right. \\
\cdot V_{\mathrm{s}}-\left(1+\frac{h}{2}\right) H(\gamma) \cdot d^{-1 / 2}
\end{gathered}
$$

avec

$$
h=\left(d-d_{0}\right) / l .
$$

Cette formule s'avère inadéquate pour rendre compte des phénomènes observés dans des couches de sulfure de plus de $460 \AA$ d'épaisseur, et une autre formulation paraît devoir être proposée, sur laquelle nous reviendrons.

\section{CONCLUSION}

Si la transition d'électrons photoexcités de la bande de valence à la bande de conduction du diélectrique détermine qualitativement les phénomènes photo- voltaiques induits par un rayonnement lumineux de plus de 3,66 eV d'énergie dans une structure sandwich $\mathrm{Al} / \mathrm{ZnS} / \mathrm{Au}$, la présence de centres coulombiens intervient pour déterminer le niveau de la tension photovoltaique, en circuit ouvert, qui croît avec le niveau du flux lumineux et avec l'épaisseur du diélectrique, entre $240 \AA$ et $460 \AA$; une quasi-bande de conduction du $\mathrm{ZnS}$ est localement créée par cumuls d'abaissements de barrière, dûs à l'ionisation des centres coulombiens illuminés; le modèle proposé permet une formulation théorique en bonne cohérence avec l'expérience.

\section{BIBLIOGRAPHIE}

1. H. Murray et A. Tosser, Thin Solid Films, 24, 165-180 (1974).

2. H. Murray et A. Tosser, Electron. Lett., 9, 491-493 (1973).

3. Handbook of Chemistry and Physics, Chemicals Rubber Co, Cleveland, Ohio, U.S.A. (1967-68).

4. H. Murray et A. Tosser, Thin Solid Films, 22, 37-44, (1974).

5. R. M. Hill, Phil. Mag., 23, 59-86, (1971).

6. J. G. Simmons, J. Appl. Phys., 34, 2581-2590, (1963).

7. H. Murray, Thèse Doct. Sc., Université de Rouen, France, (1974).

8. B. Laville St-Martin et G. Perny, Thin Solid Films, 4, 319-331, (1969). B. Laville St-Martin, Thin Solid Films, 5-6, 165-185, 359-377, (1970).

9. A. Tosser et P. Thureau, J. Phys., 28, 642-652, (1967).

10. L. Eckertova and J. Bocek, Thin Solid Films, 13, 237-241, (1972).

11. G. Dittmer, Thin Solid Films, 9, 141-172, (1972). 

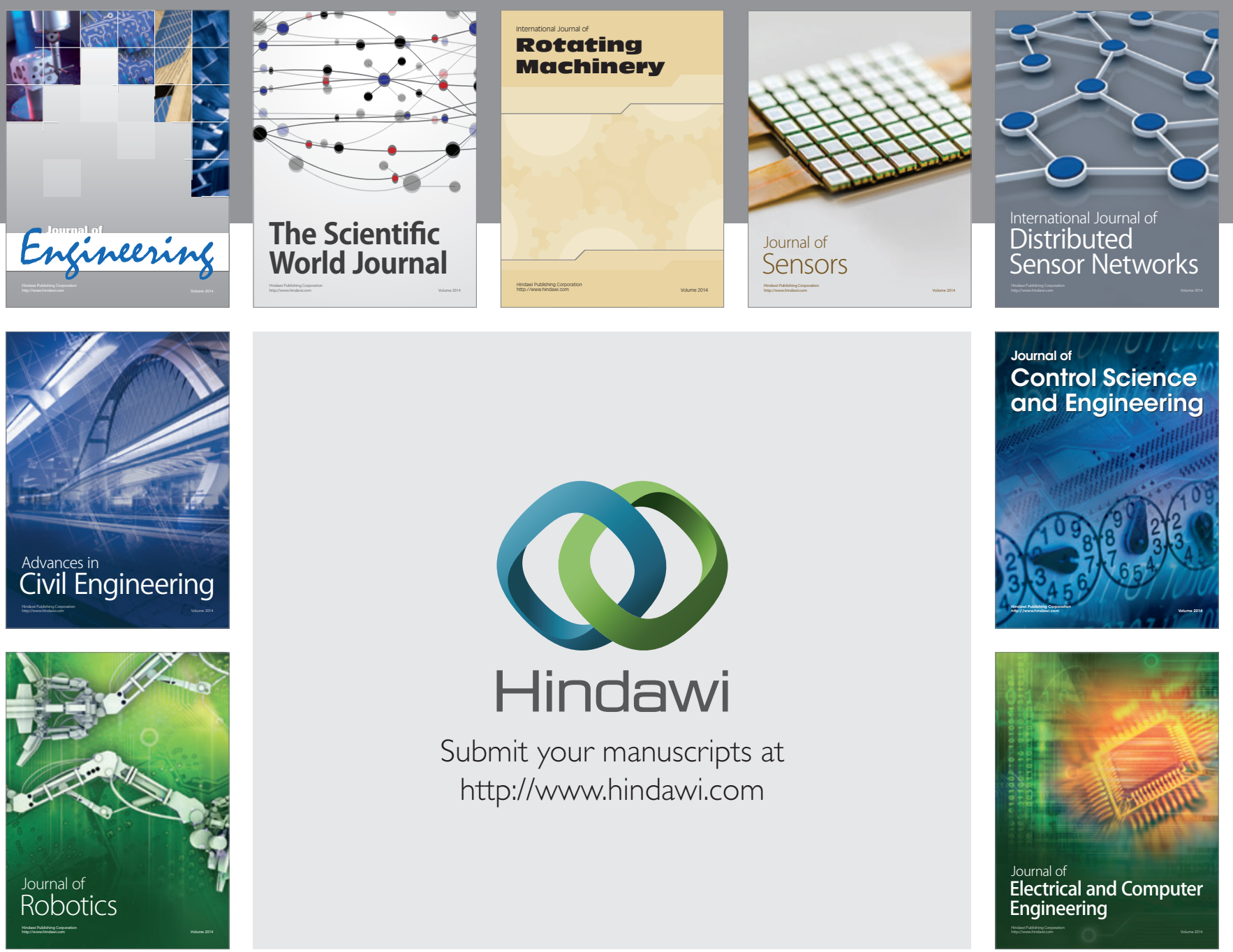

Submit your manuscripts at

http://www.hindawi.com
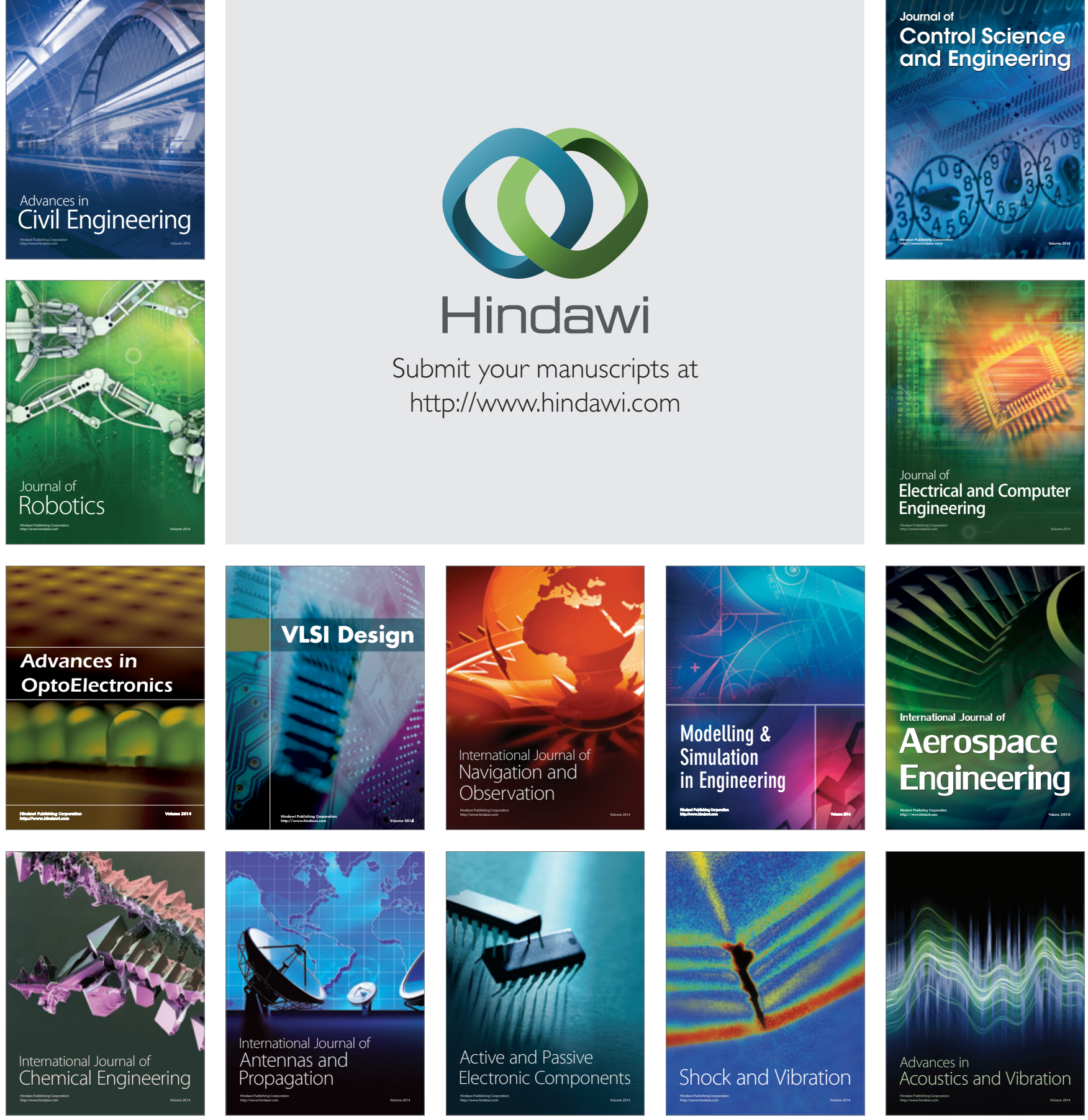